\title{
Treatment Patterns and Healthcare Costs among U.S. Patients with Advanced Melanoma Initiating Subsequent Systemic Therapy Following Use of Ipilimumab (IPI)
}

\author{
Elisabetta Malangone-Monaco ${ }^{1}$, Tony Okoro², Beata Korytowsky², Amy Stanford ${ }^{2}$, \\ Stephen Johnston ${ }^{1}$, William Johnson ${ }^{1}$, Sigrun Hallmeyer ${ }^{3}$ \\ ${ }^{1}$ Truven Health Analytics, Bethesda, USA \\ ${ }^{2}$ Bristol-Myers Squibb, Princeton, USA \\ ${ }^{3}$ Oncology Specialists, SC, Park Ridge, USA \\ Email: Elisabetta.monaco@truvenhealth.com
}

Received 8 April 2016; accepted 8 May 2016; published 11 May 2016

Copyright (C) 2016 by authors and Scientific Research Publishing Inc.

This work is licensed under the Creative Commons Attribution International License (CC BY). http://creativecommons.org/licenses/by/4.0/

c) (i) Open Access

\begin{abstract}
As the treatment landscape for advanced melanoma continues to evolve, it is critical to focus on unmet needs and understand the cost of therapy. While Ipilimumab (IPI), an immunotherapy indicated for unresectable advanced melanoma, has been a mainstay of $1^{\text {st }}$-line treatment, there was no standard of care following progression until recently. The objective of this study was to examine real-world treatment patterns and healthcare costs following IPI use in advanced melanoma patients prior to the anti-PD-1 class approval. Adult stage III or IV melanoma patients treated with IPI were selected between April 1, 2011, and September 30, 2013, from a large U.S. commercial and Medicare claims database. Patients were evaluated for therapy after IPI, with an index date set as the first systemic therapy after IPI. Per-Patient Per-Month (PPPM) healthcare costs while on active treatment were evaluated from index until treatment discontinuation, inpatient death, end of insurance enrollment, or September 30, 2013. Of 361 eligible patients, 111 (30.7\%) initiated subsequent systemic therapy (mean age, 57 years; $64.9 \%$ male). The most common therapies, single-agent or combination, included vemurafenib $(32.4 \%)$, paclitaxel $(28.8 \%)$, temozolomide $(20.7 \%)$, and carboplatin (17.1\%). During a median follow-up of 130 days, mean [standard deviation] PPPM all-cause total healthcare costs were $\$ \mathbf{2 0 , 3 8 3}[\mathbf{\$ 1 8 , 9 8 8}]$, of which $\mathbf{\$ 4 8 0 0}$ $(23.6 \%)$, $\$ 5899$ (28.9\%), and $\$ 9684(47.5 \%)$ were related to melanoma drug costs, medical claims with a diagnosis of melanoma, and other (non-specified) utilization, respectively. When considering total care, the costs of U.S. patients with advanced melanoma post-IPI were substantial across all commonly used agents.
\end{abstract}




\section{Keywords}

\section{Healthcare Costs, Ipilimumab, Melanoma, Treatment Patterns}

\section{Introduction}

Melanoma is a malignancy of melanocytes, and although it accounts for less than $2 \%$ of all skin cancers, it is responsible for the overwhelming majority of skin cancer-related deaths [1]. In the U.S., melanoma is the fifth leading cancer among men and seventh among women, and caused an estimated 9940 deaths in 2015 [1]. Melanoma is associated with excessive exposure to sunlight (i.e., ultraviolet radiation), and its main risk factors are a personal or family history of the disease and the presence of atypical, large, or numerous $(>50)$ moles. Diagnostic characteristics of a melanoma lesion include asymmetrical shape, irregular border, variable color, and a diameter greater than 6 millimeters. The treatment landscape for advanced melanoma has dramatically evolved since the 2011 U.S. Food and Drug Administration (FDA) approval of Ipilimumab (IPI), an anti-CTLA-4 immunotherapy for the treatment of unresectable or metastatic melanoma [2]. Approval of IPI was based on clinical trial findings that compared IPI with glycoprotein 100, demonstrating a significant improvement in overall survival in patients with previously treated metastatic melanoma (Kaplan-Meier estimated survival rates were $46 \%$ vs. $25 \%$ at 1 year and $24 \%$ vs. $14 \%$ at 2 years) [3]. A pooled analysis of 12 prospective and retrospective studies indicated that the overall survival rate among patients treated with IPI was $17 \%$ at 7 years with no further deaths to a maximum follow-up of 9.9 years [4].

IPI has been a mainstay of first-line treatment, but an increasing number of treatment options, such as vemurafenib, dabrafenib, trametinib, pembrolizumab, and nivolumab, approved for treatment of patients with metastatic melanoma, have since become available. Despite this changing treatment landscape and output from numerous clinical trials, few studies have focused on real-world treatment patterns. One study examined treatment patterns in metastatic melanoma patients between 2005 and 2010 (prior to the approval of IPI), and found that only $38.7 \%$ of patients received systemic therapy following diagnosis [5]. Additionally, a review article published in 2013 stressed that although there are a number of novel therapies available, these treatments are not without their limitations including some patients who will not benefit [6]. Thus there is a need to understand the post-IPI setting.

An estimated \$2.36 billion was spent in the U.S. for the treatment of melanoma in 2010, which is expected to increase to $\$ 3.16$ billion in 2020, assuming flat incidence and survival trends [7]. However, the treatment cost has increased due to the rising expenditures and extended survival offered by newly approved agents. Assuming a $2 \%$ annual cost increase in all phases of care, the burden of melanoma will rise to \$4.6 billion in 2020 [6]. While melanoma has low initial costs, the cost of treating advanced melanoma is substantial. A previous realworld analysis of patients with advanced (stage IV) melanoma reported mean Per-Patient Per-Month (PPPM) all-cause total healthcare costs of $\$ 24,844$ following diagnosis of metastatic melanoma [8]; however, little is known about the healthcare costs following the use of IPI. With the increased rate of melanoma and the rising costs, it has become even more critical to focus on unmet needs and understand the associated cost of treatment.

The objective of this study was to use administrative claims data to examine real-world treatment patterns and healthcare costs following IPI use in patients with advanced melanoma.

\section{Materials and Methods}

De-identified U.S. administrative claims data from the Truven Health Analytics MarketScan ${ }^{\circledR}$ Commercial Claims and Encounters and Medicare Supplemental Databases from 2011-2013 were used with follow-up through September 30, 2013. These databases contain inpatient medical, outpatient medical, and outpatient prescription drug data for individuals with employer-sponsored primary or Medicare supplemental insurance. In 2012, the MarketScan Commercial Claims and Encounters Database contained the inpatient, outpatient, and outpatient prescription drug experience of approximately 31.3 million employees and their dependents. The MarketScan Medicare Supplemental and Coordination of Benefits (COB) Database contained the healthcare experience of approximately 3.1 million retirees with Medicare supplemental insurance paid for by employers. As a retrospective, administrative claims database study, informed consent and ethics committee review were not required. 


\subsection{Study Design and Patient Selection Criteria}

This study used a retrospective, observational cohort design (Figure 1). Patients selected for this study were required to have a diagnosis of a secondary malignant neoplasm (ICD-9-CM diagnosis codes 196.xx, 197.xx, 198.xx) on $\geq 1$ non-diagnostic medical claim occurring between April 1, 2011 and September 30, 2013 (the secondary malignant neoplasm date corresponded to the earliest claim for a secondary malignant neoplasm during this period). For patients with stage III disease, a diagnosis of melanoma (ICD-9-CM diagnosis codes 172.xx) on $\geq 1$ inpatient or $\geq 2$ non-diagnostic outpatient medical claims (on different days) occurring in the 180 days before the secondary malignant neoplasm date was required. Patients were defined as having stage IV disease if they met the above criteria and additionally presented with a claim for a skin biopsy or removal of skin lesion procedure in the 180 days before their secondary malignant neoplasm date with a diagnosis of melanoma on any inpatient or outpatient medical claim within 30 days of the date of the procedure.

Patients were excluded if they had a diagnosis for a non-melanoma primary cancer occurring on, or during 180 days prior to the secondary malignant neoplasm date unless 1 ) the other primary cancer was skin cancer or a hematologic malignancy OR 2) the patient had a claim for melanoma on the diagnosis date OR 3) the site of the reported other cancer was the same as the site of metastasis.

Since the study's intent was to evaluate a post-IPI period, patients were required to have a new initiation of IPI following the diagnosis of melanoma. The IPI completion date corresponded to the last observed IPI claim + 21 days. Additionally, patients received subsequent chemotherapy, targeted therapy, or immunotherapy after the IPI completion date (first claim set as index date). Patients were required to have at least 6 months of continuous enrollment with pharmacy benefits before the IPI completion date (baseline period), and patients under 18 were excluded from the study sample.

\subsection{Study Measures and Statistical Analyses}

Patient demographics were collected at index date, and clinical characteristics were measured throughout the baseline period. Beginning on the index date, patients were followed to measure the time to receipt of additional therapy after IPI, the number of and the specific therapies received in the first regimen after IPI, and the time to discontinuation ( $\geq 60$-day gap in therapy). Univariate descriptive statistics were used to analyze the study measures, and for all time-to-event outcomes, censoring of person time occurred at the earliest of inpatient death, end

Study period: April 1, 2011 - September 30, 2013

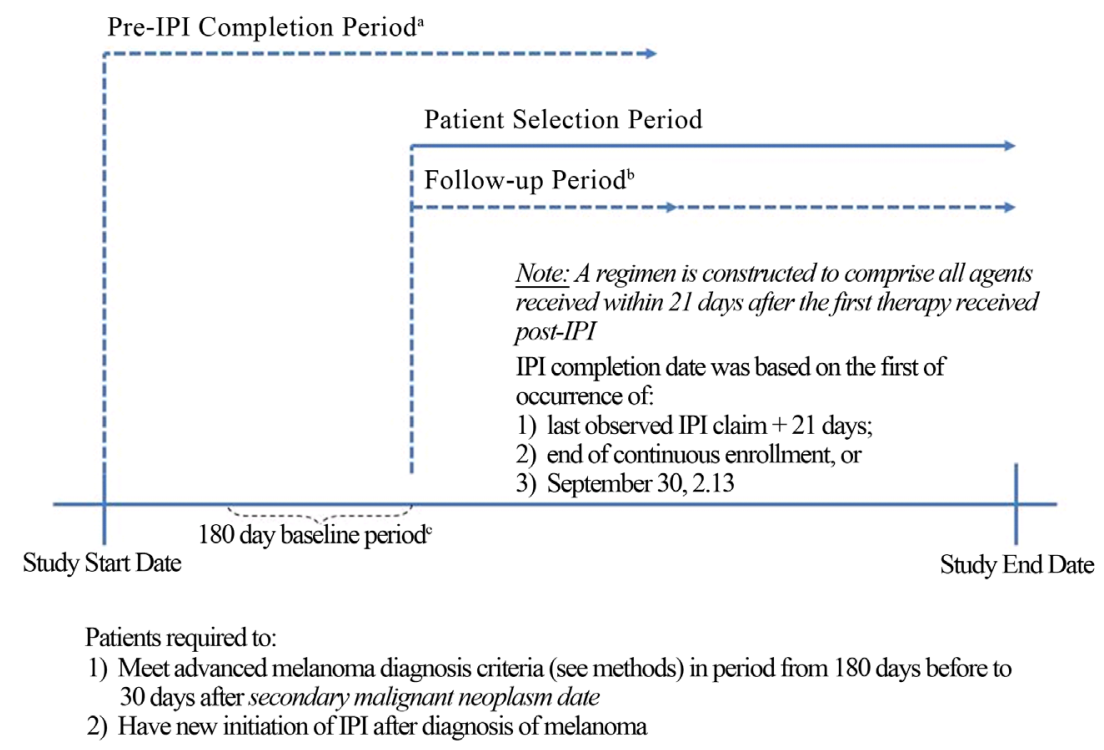

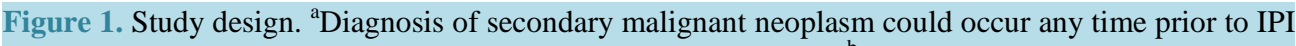
completion date between April 1, 2011 and September 30, 2013. ${ }^{b}$ Patients were followed forward in time from first post-IPI therapy until its discontinuation or censoring at end of continuous enrollment or September 30, 2013. 'Baseline period was defined as the 180 days prior to IPI completion date. 
of insurance enrollment, or September 30, 2013. The Kaplan Meier [9] method of survival analysis was used to visually depict the distribution of time to each outcome.

All cost analyses were evaluated per-patient per-month (PPPM) due to variable length of follow-up. PPPM healthcare costs were computed as (total healthcare costs/total follow-up) $\times 30$. PPPM all-cause total healthcare costs while on active treatment were evaluated from the index date until a 60-day gap in treatment, inpatient death, end of insurance enrollment, or September 30, 2013 (follow-up period). Healthcare costs include the gross covered payments for the service or product. PPPM all-cause total healthcare costs were subdivided into melanoma systemic therapy drug costs (office-administered and pharmacy costs), medical claims with a diagnosis of melanoma, and all other (non-specified) utilization. Costs and utilization were measured for inpatient (hospitalizations), outpatient (emergency department visits, outpatient physician office visits, radiation services, outpatient surgical procedures, and outpatient services), pharmacy costs (excluding systemic therapy for advanced melanoma), and pharmacy and medical costs for systemic therapy for advanced melanoma, including administration of infused medications.

\section{Results}

Among a total of 4,522 patients with advanced melanoma and no other primacy cancer, 640 had an IPI claim following their diagnosis, and 361 patients met continuous enrollment and age requirements. Over a mean [standard deviation (SD), median] post-IPI completion follow-up of 141 [142, 96] days, a total of 111 patients (31\% of 361) received systemic therapy post-IPI. Table 1 displays the demographic and clinical characteristics of the 111 patients. The population was on average 57 years old and $65 \%$ male. During the 6 -month baseline period prior to the index date, $30.2 \%$ of patients received a treatment recommended per 2013 NCCN Guidelines ${ }^{\circledR}$, including non-IPI immunotherapy (13.0\%), chemotherapy (11.6\%), and/or targeted therapy (10.8\%). Among the 111 patients who received therapy post-IPI, mean [SD, median] time from the IPI completion date to the index date was $85[74,63]$ days. The three most common therapies received in the first regimen post-IPI were vemurafenib (32.4\%), paclitaxel (28.8\%), and temozolomide (20.7\%) (Figure 2(a)). Overall, 77.5\% of patients received a single agent in their first regimen post-IPI, 18.9\% received two agents, and 3.6\% received three or more agents (Figure 2(b)). A total of 22 (19.8\%) patients received carboplatin and paclitaxel with or without other agents. Additionally, a total of 7 (6.3\%) patients discontinued therapy post-IPI over a mean [SD, median] follow-up of 169 [114, 130] days. Four of these patients received further treatment with a $2^{\text {nd }}$ subsequent line of therapy post-IPI. Among those observed to discontinue therapy post-IPI, the mean [SD, median] time-to discontinuing therapy post-IPI was $157[96,128]$ days.

Figure 3(a) displays the components of the PPPM all-cause total healthcare costs while on active treatment related to melanoma drugs, medical claims with a diagnosis of melanoma, and other (non-specified) utilization. During median post-index follow-up of 130 days, patients incurred mean [standard deviation, SD] PPPM allcause total healthcare costs of $\$ 20,383$ [ $\$ 18,988$ ], of which $24 \%$ was related to melanoma drug costs, $29 \%$ was related to medical claims with a diagnosis of melanoma, and $48 \%$ was related to other (non-specified) utilization. Figure 3(b) displays the PPPM all-cause total healthcare costs incurred for the first regimen post-IPI during follow-up, specifically among the four systemic therapies that were used most commonly after IPI completion; these therapies could be used as single agent or in combination with one another, meaning the groups were not mutually exclusive. Whereas melanoma drug costs were numerically lowest for paclitaxel and carboplatin, other (nonspecified) costs associated with the use of these therapies were numerically greatest. The proportion of PPPM all-cause total healthcare costs related to other (non-specified) utilization ranged from $36 \%$ for patients treated with vemurafenib to $74 \%$ for patients treated with carboplatin.

\section{Discussion}

One third of study patients were treated following initial IPI use. This highlights the unmet need after IPI therapy during the time the study was conducted (2011-2013). Treatment for advanced melanoma after IPI has since evolved, but there is little research in this post-IPI setting to illustrate the best treatment for patients following progression while treated with IPI. Since the conduct of our study, several treatment options have been approved by the FDA, including additional treatments that inhibit mutated BRAF proteins in the mitogen activated protein kinase (MAPK) pathway and the MEK protein [10] as well as immune checkpoint blocking agents such as PD-1 and PD-L1 antibodies [11]. 
Table 1. Patient baseline demographic and clinical characteristics (treated patients, $\mathrm{N}=111$ ).

Patients with post-IPI therapy $\mathrm{N}=111$

\section{Demographic characteristics measured at index}

Age, mean (SD)

Male, \%

Medicare beneficiary, \%

Insurance plan type, \%

Comprehensive

EPO

HMO

POS (includes w/capitation)

PPO

Other (basic/major medical, CHDP, HDHP)

Unknown/missing

Clinical characteristics measured at index and during baseline period

Baseline National Cancer Institute comorbidity index, mean (SD)

Baseline unique number of National Drug Codes, mean (SD)

Baseline selected comorbid conditions with $>5 \%$ prevalence, $\%$

Brain/leptomeningeal metastasis

Hypertension

Chronic liver disease

Myocardial infarction/coronary artery disease

Diabetes

Cerebrovascular disease

Renal disease

Autoimmune disorders

Congestive heart failure

Ocular melanoma

Diagnosing physician specialty, \%

Oncologist/hematologist

General surgeon

Dermatologist

Primary care provider

Radiologist/nuclear medicine

Other specialist

Other provider

Facility

Unknown

8.1

$\mathrm{CDHP}=$ consumer-directed health plan; $\mathrm{EPO}=$ exclusive provider organization; $\mathrm{HDHP}=$ high-deductible health plan; HMO = health maintenance organization; $\mathrm{POS}$ = point of service; $\mathrm{PPO}$ = preferred provider organization; $\mathrm{SD}=$ standard deviation . 
(a) Specific therapies received in first regimen post-IPI

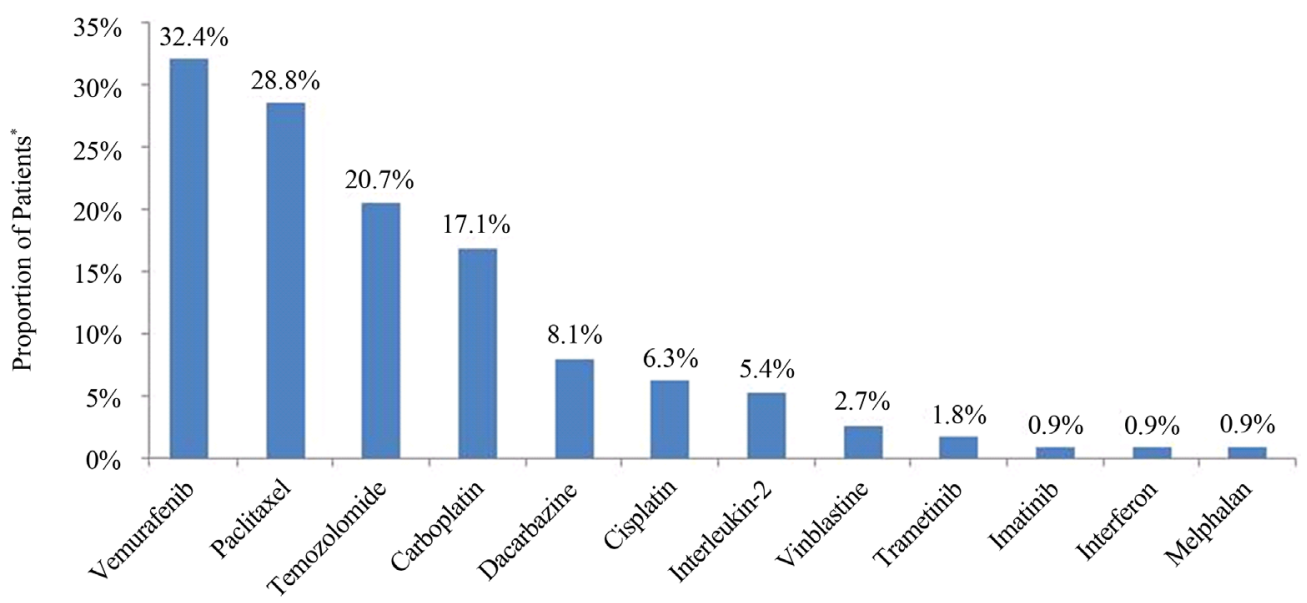

(b) Number of therapies received in first regimen post-IPI

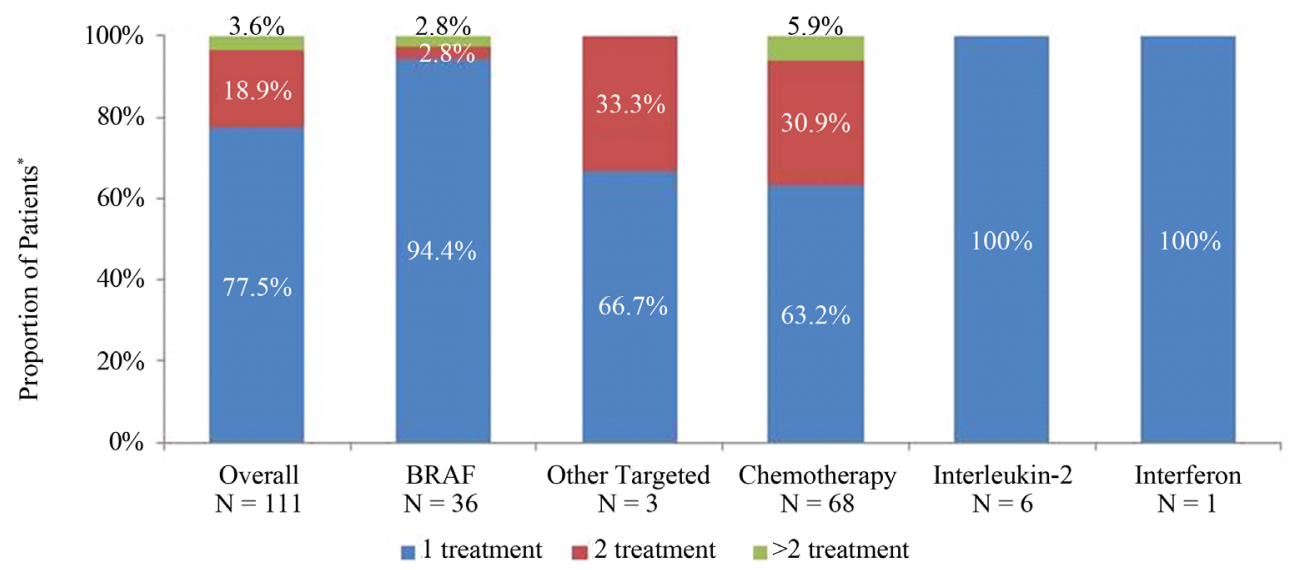

Figure 2. Therapies received post-IPI. *Agents could be used as monotherapy or in combination with others; no patients were observed to receive dabrafenib; therapies were received over a mean [SD, median] follow-up of 141 [142, 96] days post-IPI.

Dabrafenib [12] and trametinib [13], manufactured by GlaxoSmithKline, were approved first as single agents in May 2013 and then in combination [14] in January 2014, for patients with unresectable or metastatic melanoma with a BRAF V600E or V600K mutation. Dabrafenib and trametinib were offered in capsule and tablet form, respectively. Later in September 2014, pembrolizumab [15], manufactured by Merck, was approved in this same unresectable or metastatic melanoma population, following progression with IPI treatment, and a BRAF inhibitor if BRAF V600 mutation positive. Most recently, nivolumab [16], manufactured by BristolMyers Squibb, was approved for patients with unresectable or metastatic melanoma who present with disease progression following treatment with IPI and, if BRAF V600 mutation positive, a BRAF inhibitor. Ongoing studies have demonstrated the benefit of the PD-1 and PD-L1 class in the treatment-naïve setting and FDA approval is pending. Our study provides valuable information with regards to the post-IPI setting, but it will be important to revisit this study during the time period when these additional therapies become available to assess how treatment patterns and outcomes evolve for advanced melanoma.

The study results also illustrate the substantial and variable cost burden that advanced melanoma imposes. In our study, all-cause PPPM costs were mean [SD] \$20,383 [\$18,988]. This compares to mean [SD] PPPM costs of $\$ 10,999$ [\$11,319] and $\$ 15,762$ [\$12,377] for patients with 1 to 3 and 4 or more metastases, respectively [17]. According to Guy et al. [18], the annual cost of treating melanoma in the U.S. is $\$ 3.3$ billion.

\section{Limitations}

The study had limitations inherent with administrative claims databases. Administrative claims data are 
(a) PPPM all-cause total health care cost components during follow-up

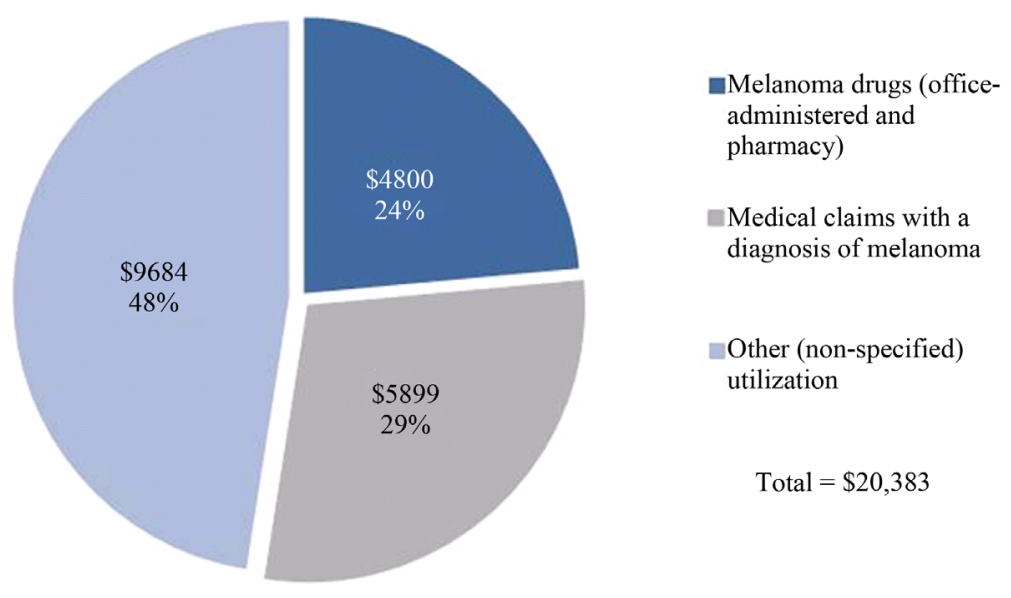

(b) PPPM all-cause total health care costs incurred during follow-up

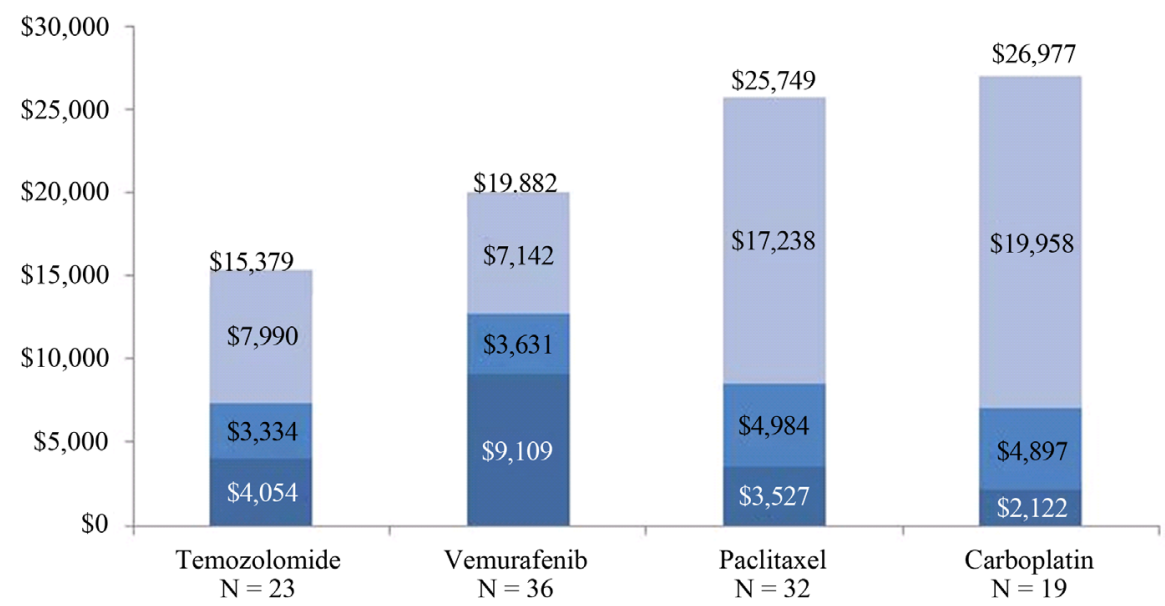

घelanoma drugs $\square$ Medical claims with a diagnosis of melanoma $\quad$ Other (non-specified) utilization

Figure 3. PPPM all-cause total healthcare costs incurred during follow-up.

generated for the purposes of provider reimbursement and not for analysis. As such, information derived from medical billing codes may be subject to omissions or errors and may lead to measurement error. This study represents the real-world practice patterns for advanced melanoma patients, but is limited to individuals with employer-sponsored health coverage and/or Medicare supplemental coverage. Consequently, findings from this study may not be representative of the entire U.S.

Patients were followed for as long as possible after treatment with IPI to ascertain study outcomes, but patients may be lost to follow-up when they switch employers. It is also unknown whether patients who were observed to receive no additional therapy post-IPI went on to receive therapy after the window of observation. Analyses of data with longer follow-up and larger samples are warranted.

Aside from database limitations, the identification of claims for IPI delivered in the outpatient setting was subject to the availability of a unique $\mathrm{J}$ code for the medication. In the time period between the introduction of IPI and the creation of a unique code, J9999 was used to identify these claims. As such, some patients may have incorrectly been assigned as having received IPI treatment. In addition, the specific line of therapy to which the use of IPI, or subsequent therapy post-IPI, corresponded has not been established. The category of other 
(non-specified) utilization may represent some services that were provided for the care of advanced melanoma but not coded as such. Finally, the study results are reflective of the period between April 2011 and September 2013, a time since which treatment patterns and healthcare costs have changed.

\section{Conclusions}

The treatment of patients with advanced melanoma was considerably altered with the approval of IPI, which has become a mainstream first-line therapy. Although recently approved agents for patients with advanced melanoma may provide additional benefit, many physicians revert to treating patients with traditional chemotherapies, especially for patients who progress. These data highlight the limited use and lack of a standard of care in the post-IPI setting prior to September 2013 and demonstrate a degree of unmet need that existed for patients who exhausted their options.

When considering the totality of care, including management beyond systemic therapy alone, the costs of U.S. patients with advanced melanoma after being treated with IPI were substantial across all commonly used agents. Considering the long-term costs when determining treatment for advanced melanoma patients may have important economic ramifications. This real-world treatment analysis should be revisited when longer-term data are available following IPI to assess the continually changing landscape and entry of newer treatment options.

\section{Acknowledgements}

The authors would like to acknowledge Donna McMorrow for her contributions.

\section{References}

[1] American Cancer Society (2015) Cancer Facts \& Figures 2015. American Cancer Society, Atlanta.

[2] Salama, A.K. (2013) Evolving Pharmacotherapies for the Treatment of Metastatic Melanoma. Clinical Medicine Insights: Oncology, 7, 137-149. http://dx.doi.org/10.4137/CMO.S9565

[3] Hodi, F.S., O’Day, S.J., McDermott, D.F., et al. (2010) Improved Survival with Ipilimumab in Patients with Metastatic Melanoma. New England Journal of Medicine, 363, 711-723. http://dx.doi.org/10.1056/NEJMoa1003466

[4] Schadendorf, D., Hodi, F.S., Robert, C., et al. (2015) Pooled Analysis of Long-Term Survival Data from Phase II and Phase III Trials of Ipilimumab in Unresectable or Metastatic Melanoma. Journal of Clinical Oncology, 33, 1889-1894. http://dx.doi.org/10.1200/JCO.2014.56.2736

[5] Zhao, Z., Wang, S. and Barber, B.L. (2014) Treatment Patterns in Patients with Metastatic Melanoma: A Retrospective Analysis. Journal of Skin Cancer, 2014, Article ID: 371326. http://dx.doi.org/10.1155/2014/371326

[6] Menaa, F. (2013) Latest Approved Therapies for Metastatic Melanoma: What Comes Next? Journal of Skin Cancer, 2013, Article ID: 735282. http://dx.doi.org/10.1155/2013/735282

[7] Mariotto, A.B., Robin, Y.K., Shao, Y., et al. (2011) Projections of the Cost of Cancer Care in the United States: 20102020. Journal of the National Cancer Institute, 103, 117-128. http://dx.doi.org/10.1093/jnci/djq495

[8] Malangone, E., Hirji, I., Hallmeyer, S., Meyer, N., Rao, A.S. and Purdum, A.G. (2014) Trends in US Treatment Patterns and Total Health Care Expenditures of Newly Diagnosed Advanced Melanoma Patients from a Medicare and Commercially Insured Database, 2011-2013. Poster Presented at the Academy of Managed Care Pharmacy 2014 Nexus Meeting, Boston, 7-10 October 2014.

[9] Kaplan, E.L. and Meier, P. (1958) Nonparametric Estimation from Incomplete Observations. Journal of the American Statistical Association, 53, 457-481. http://dx.doi.org/10.1080/01621459.1958.10501452

[10] Olszanski, A.J. (2014) Current and Future Roles of Targeted Therapy and Immunotherapy in Advanced Melanoma. Journal of Managed Care \& Specialty Pharmacy, 20, 346-356. http://dx.doi.org/10.18553/jmcp.2014.20.4.346

[11] Berberabe, T. (2014) The Evolving Treatment Landscape in Advanced Melanoma. http://www.onclive.com/publications/oncology-live/2014/december-2014/the-evolving-treatment-landscape-in-advanc ed-melanoma/2

[12] Dabrafenib (2015) U.S. Food and Drug Administration. http://www.fda.gov/Drugs/InformationOnDrugs/ApprovedDrugs/ucm354477.htm

[13] Dabrafenib (2015) U.S. Food and Drug Administration. http://www.fda.gov/Drugs/InformationOnDrugs/ApprovedDrugs/ucm354478.htm

[14] Trametinib and Dabrafenib (2015) U.S. Food and Drug Administration. http://www.fda.gov/Drugs/InformationOnDrugs/ApprovedDrugs/ucm381451.htm 
[15] Pembrolizumab (2015) U.S. Food and Drug Administration. http://www.fda.gov/Drugs/InformationOnDrugs/ApprovedDrugs/ucm412861.htm

[16] Nivolumab (2015) U.S. Food and Drug Administration. http://www.fda.gov/Drugs/InformationOnDrugs/ApprovedDrugs/ucm427807.htm

[17] Reyes, C., Byfield, S.D., Linke, R., et al. (2013) The Burden of Metastatic Melanoma: Treatment Patterns, Healthcare Use (Utilization), and Costs. Melanoma Research, 23, 160-166. http://dx.doi.org/10.1097/CMR.0b013e32835e58d6

[18] Guy, G.P., Machlin, S.R., Ekwueme, D.U., et al. (2015) Prevalence and Costs of Skin Cancer Treatment in the US, 2002-2006 and 2007-2011. American Journal of Preventive Medicine, 48, 183-187. http://dx.doi.org/10.1016/j.amepre.2014.08.036

\author{
Abbreviations \\ CDHP = consumer-directed health plan; \\ $\mathrm{COB}=$ Coordination of Benefits; \\ $\mathrm{EPO}=$ exclusive provider organization; \\ FDA = Food and Drug Administration; \\ HDHP = high-deductible health plan; \\ $\mathrm{HMO}=$ health maintenance organization; \\ IPI = ipilimumab; \\ MAPK = mitogen activated protein kinase; \\ $\mathrm{POS}=$ point of service; \\ $\mathrm{PPO}=$ preferred provider organization; \\ PPPM = per-patient per-month; \\ $\mathrm{SD}=$ standard deviation
}

